Proyecciones Journal of Mathematics

Vol. 28, Nº 3, pp. 209-226, December 2009.

Universidad Católica del Norte

Antofagasta - Chile

\title{
CONNECTEDNESS IN JÄGER - ŠOSTAK'S I-FUZZY TOPOLOGICAL SPACES *
}

\author{
FANG JINMING \\ and \\ GUO YUANMEI \\ OCEAN UNIVERSITY OF CHINA, CHINA \\ Received : January 2009. Accepted : June 2009
}

\begin{abstract}
G.Jäger [Compactness and connectedness as absolute properties in fuzzy topological spaces, Fuzzy sets and Systems 94(1998) 405-401] introduced a kind of (general) fuzzy topological space. In this paper, we propose a new kind of topological space in Šostak's sense, called JägerŠostak's I-fuzzy topological space, which reduced to Jäger's (general) fuzzy topological to two-valued logic. After that for each fuzzy subset of Jäger-Šstak's I-fuzzy topological space, we define a degree of connectedness, which overcome the deficit of study for the whole space a degree of being connected in public papers, and establish two characteristic theorems of the degree of being connectedness. Doing so we find that the degree of connectedness is an absolute property in JägerŠostak's I-fuzzy topology.
\end{abstract}

Keywords: Jäger-Šostak's I-fuzzy topology; subspace; the degree of connectedness; absolute property

${ }^{*}$ This work is supported by the National Natural Science Foundation of PR China (No. 10771200) 


\section{Introduction and preliminaries}

Since C.L. Chang [1] introduced fuzzy theory into topology, many authors discussed various aspects of fuzzy ( $I$-fuzzy, fuzzifying) topology. For connectedness of fuzzy topological space, many authors devoted their researches to the topics, such as $[4,6,11]$. In [6], the author proposed a concept of connectedness and showed it is an absolute property in (general) fuzzy topology space. Now we'll study degree of connectedness of Jäger-Šostak's $I$-fuzzy topological space in more general form.

The contents are arranged as follows. In next section, we introduce basic concepts, such as Jäger-Šostak's I-fuzzy topology space, morphisms and its degree of continuity. Moreover, we give the definition of Jäger-Šsostak's $I$-fuzzy subspace, which is the crucial part of this paper so that the degree of connectedness defined in the paper is an absolute property. In last section, for each fuzzy subset of Jäger-Šstak's I-fuzzy topological space, we introduce a degree of connectedness and show its characteristic theorem of the degree of being connectedness. After that, we give another characteristic theorem of the degree of connectedness by the degree of separation. Finally, we conclude that the degree of connectedness defined in the paper, is an absolute property in Jäger-Šsostak's $I$-fuzzy topology.

In this paper, $X, Y, \cdots$ are nonempty sets. A fuzzy subset on a universal set $X$ is a mapping from $X$ to the real unit interval [0,1]. All fuzzy subsets on $X$ is denoted by $I^{X}$ and for a subset $Y \subseteq X$, the characteristic function of $Y$ is denoted by $1_{Y}$. Fuzzy subsets are denoted by capital letters $A, B, C, \cdots \in[0,1]^{X}$. And the greatest element of $I^{X}$ is denoted by $1_{X}$ and the least element of $I^{X}$ is denoted by $0_{X}$. Note that we don't distinguish a number $\lambda \in[0,1]$ and the constant function $\lambda: X \rightarrow[0,1]$ such that $\lambda(x)=\lambda$ for all $x \in X$. It's assumed that the reader is acquired with the usual definitions and notations in fuzzy set theory and fuzzy topology. Given a fuzzy subset $A \in[0,1]^{X}$, we denote $\mathcal{F}_{X}(A):=\left\{B \in[0,1]^{X}: B \leq A\right\}$. We call $A_{0}:=\{x \in X: A(x)>0\}$ the support of $A$, and identify $\mathcal{F}_{X}(A)$ with $\mathcal{F}_{A_{0}}(A)$. If $B \in \mathcal{F}_{X}(A),\left\langle{ }^{A} B\right.$, the complement of $B$ relative to $A$, is defined via $\left\langle{ }^{A} B(x):=A(x)-B(x)\right.$ for all $x \in X$. For the complement of $B$ relative $A$, we have following proposition to describe its basic properties and omit their proofs.

Proposition 1.1. For any fuzzy subset $A \in I^{X}$, the following hold:

(1) For each $B \in \mathcal{F}_{X}(A),\left\langle{ }^{A}\left\langle{ }^{A} B=B\right.\right.$;

(2) For all $B, C \in \mathcal{F}_{X}(A), B \leq C \Rightarrow\left\langle{ }^{A} C \leq\left\langle{ }^{A} B\right.\right.$; 
(3) For all $B, C \in \mathcal{F}_{X}(A),\left\langle{ }^{A}(B \wedge C)=\left\langle{ }^{A} B \vee\left\langle{ }^{A} C\right.\right.\right.$ and $\left\langle{ }^{A}(B \vee C)=\right.$ $\left\langle{ }^{A} B \wedge\left\langle{ }^{A} C\right.\right.$.

\section{Jäger-Šostak's $I$-fuzzy topological spaces}

Jäger [6] proposed a concept of a fuzzy topology on a fuzzy subsets $A$ so that a lot of topological questions can be researched on any fuzzy subsets in place of a whole space. According to the ideas of $L$-fuzzy topology in the sense of [5], we generalize Jäger's fuzzy topology to a fuzzy subset as follows.

Definition 2.1. $\quad$ Let $A \in[0,1]^{X}$. A map $\tau: \mathcal{F}_{X}(A) \rightarrow[0,1]$ is called a Jäger-Šostak's $I$-fuzzy topology on a fuzzy subset $A$ iff $\tau$ satisfies the following conditions:

(JSO1) $\tau\left(\left\langle{ }^{A}(A \wedge \alpha)\right)=1\right.$ for all $\alpha \in[0,1]$,

$(\mathrm{JSO} 2) \tau(G \wedge H) \geq \tau(G) \wedge \tau(H)$ for every $G, H \in \mathcal{F}_{X}(A)$,

(JSO3) $\tau\left(\bigvee_{J \in J} A_{j}\right) \geq \bigwedge_{j \in J} \tau\left(A_{j}\right)$ for each family $\left\{A_{j}: j \in J\right\} \subseteq$ $\mathcal{F}_{X}(A)$.

The pair $(A, \tau)$ is called a Jäger-Šostak's $I$-fuzzy topological space (JSI$\mathrm{fts}$, in short). The value of $\tau(B)$ for every $B \in \mathcal{F}_{X}(A)$ is interpreted as the degree to which B is open relative to $\tau$, the value of $\tau\left(\left\langle{ }^{A} H\right)\right.$ for each $H \in \mathcal{F}_{X}(A)$ is the degree to which $\mathrm{H}$ is closed relative to $\tau$.

Remarks 2.2. Assume that $\tau: \mathcal{F}_{X}(A) \rightarrow I$ is a Jäger-Šostak's $I$-fuzzy topology. Then a (general) fuzzy topology $\mathcal{I}_{\tau}$ on the fuzzy subset $A$ in the sense of [6] can be induced by $\tau$, that is, $\mathcal{T}_{\tau}=\{U: \tau(U)=1\}$ satisfies the following conditions:

(o1) $\left\langle{ }^{A}(A \wedge \alpha) \in \mathcal{T}_{\tau}\right.$ for all $\alpha \in[0,1]$,

(o2) $G, H \in \mathcal{T}_{\tau} \Rightarrow G \wedge H \in \mathcal{T}_{\tau}$ for every $G, H \in \mathcal{F}_{X}(A)$,

(o3) $\forall j \in J, A_{j} \in \mathcal{T}_{\tau} \Rightarrow \bigwedge_{j \in J} A_{j} \in \mathcal{T}_{\tau}$ for each family $\left\{A_{j}: j \in J\right\} \subseteq$ $\mathcal{F}_{X}(A)$.

Conversely, if $\mathcal{T} \subseteq I^{X}$ is a general fuzzy topology, then a Jäger-Šsostak's $I$-fuzzy topology $\tau_{\mathcal{T}}$ is obtained by defining $\tau_{\mathcal{T}}(U)=1$ whenever $U \in \mathcal{T}$ and $\tau_{\mathcal{T}}(U)=0$ otherwise. Therefore, Jäger-Šostak's $I$-fuzzy topology is more general than a (general) fuzzy topology.

In order to obtain morphisms between two JSI-fts's, we have to restrict function $f: \mathrm{X} \rightarrow \mathrm{Y}$ to fuzzy subsets as Jäger [6] did. Given such a function $f$ : $\mathrm{X} \rightarrow \mathrm{Y}$ and $A \in[0,1]^{X}$, we called the mapping $g:=f \mid A: \mathcal{F}_{X}(A) \rightarrow[0,1]^{Y}$, $B \rightarrow f(B)$ the restriction of $f$ on $\mathrm{A}$, where for all $y \in Y$, 
$f(B)(y)=\sup \{B(x): f(x)=y\}$ as defined by Zadeh. The inverse imagine of a fuzzy subset $D \in[0,1]^{Y}$ under $f \mid A$ is defined by

$$
(f \mid A)^{-1}(D)=\bigvee\{C:(f \mid A)(C) \leq D\} .
$$

Moreover, we have $(f \mid A)^{-1}(D)=A \wedge f^{-1}(D)$, where $f^{-1}(D)=D \circ f$.

Definition 2.3 (Jäger [6]). We say that $g: A \rightarrow B$ is a fuzzy mapping from A to B if and only if there exists a function $f: X \rightarrow Y$ such that $f \mid A=g$ and $f(A) \leq B$.

Now we propose the definition of morphism between two JSI-fts's.

Definition 2.4. Let $(A, \tau)$ and $(B, \delta)$ be two JSI-fts's. A fuzzy mapping $g: A \rightarrow B$ is called continuous if and only if for each $H \in \mathcal{F}_{Y}(B)$

$$
\delta\left(\left\langle^{B} H\right) \leq \tau\left(\left\langle^{A}\left(g^{-1}(H)\right)\right) .\right.\right.
$$

Moreover, we would define the degree to which $g:(A, \tau) \rightarrow(B, \delta)$ is continuous using Łukasiewicz's logic.

Definition 2.5. In the situation above, for each fuzzy mapping $g:(A, \tau) \rightarrow$ $(B, \delta)$, the degree to which $\mathrm{g}$ is continuous, is defined

$$
c(g)=\bigwedge_{H \in \mathcal{F}_{Y}(B)} \min \left\{1,1-\delta\left(\left\langle^{B} H\right)+\tau\left(\left\langle{ }^{A}\left(g^{-1}(H)\right)\right)\right\} .\right.\right.
$$

Thus, a fuzzy mapping $g: A \rightarrow B$ is continuous if and only if its degree to which $g$ is continuous is 1 .

Proposition 2.6. The follow conclusions hold:

(i) Let $g=i d_{X} \mid A:(A, \tau) \rightarrow(A, \tau)$, then $\mathrm{c}(\mathrm{g})=1$,

(ii) If $g:(A, \tau) \rightarrow(B, \delta)$ and $h:(B, \delta) \rightarrow(C, \sigma)$, then $c(g) * c(h) \leq c(h \circ$ $g$ ), where $*$ is the Eukasiewicz $t$-norm and hence $c(g) * c(h)=\max \{c(g)+$ $c(h)-1,0\}$,

(iii) For the restriction of a constant function $f: X \rightarrow Y$, we have $\mathrm{c}(\mathrm{f})=1$.

Proof. (i) is obvious. In order to show (ii), we show inequality in two cases: 
Case $1, c(h) \leq c(h \circ g)$. We have

$$
\begin{aligned}
c(g) * c(h) & =\max \{c(g)+c(h)-1,0\} \\
& \leq \max \{c(g)+c(h \circ g)-1,0\} \\
& \leq c(h \circ g) .
\end{aligned}
$$

Case $2, c(h) \not \leq c(h \circ g)$. In this case, it holds that $c(h \circ g)<c(h)$. Thus

$$
\begin{aligned}
0<c(h)-c(h \circ g)= & \inf _{H \leq C} \min \left\{1,1-\sigma\left(\left\langle{ }^{C} H\right)+\delta\left(\left\langle{ }^{B}\left(h^{-1}(H)\right)\right\}\right.\right.\right. \\
& -\inf _{H \leq C} \min \left\{1,1-\sigma\left({ }^{C} H\right)+\tau\left(\left\langle^{A}(h \circ g)^{-1}(H)\right)\right\}\right. \\
\leq & \sup _{H \leq C}\left(\delta \left(\left\langle^{B}\left(h^{-1}(H)\right)\right)-\tau\left(\left\langle{ }^{A}\left(\left(g^{-1} \circ h^{-1}(H)\right)\right)\right)\right.\right.\right. \\
\leq & \sup _{G \leq B}\left(\delta\left(\left\langle{ }^{B} G\right)\right)-\tau\left(\left\langle{ }^{A} g^{-1}(G)\right) .\right.\right.
\end{aligned}
$$

It follows that

$$
\begin{aligned}
c(g) & =\inf _{G \leq B} \min \left\{1,1-\delta\left(\left\langle^{B} G\right)+\tau\left(\left\langle{ }^{A}\left(g^{-1}(G)\right)\right)\right\}\right.\right. \\
& =\min \left\{1, \inf _{G \leq B}\left(1-\delta\left(\left\langle^{B} G\right)+\tau\left(\left\langle{ }^{A} g^{-1}(G)\right)\right)\right\}\right.\right. \\
& \leq \min \{1,1-c(h)+c(h \circ g)\} \\
& =1-c(h)+c(h \circ g) .
\end{aligned}
$$

That is, $c(g)+c(h)-1 \leq c(h \circ g)$. Therefore, $c(g) * c(h) \leq c(h \circ g)$ holds.

For the proofs of (iii), note that for each $H \in \mathcal{F}_{Y}(B), f^{-1}(H)=\alpha$ and $(f \mid A)^{-1}(H)=A \wedge f^{-1}(H)=A \wedge \alpha$ for some $\alpha \in[0,1]$. Thus

$$
\begin{aligned}
c(f) & =\inf _{H \leq B} \min \left\{1,1-\sigma(H)+\tau\left(\left\langle{ }^{A}(f \mid A)^{-1}(H)\right)\right\}\right. \\
& =\inf _{H \leq B} \min \left\{1,1-\sigma(H)+\tau\left(\left\langle{ }^{A} A \wedge \alpha\right)\right\}\right. \\
& =1 . \quad \square
\end{aligned}
$$


In the following, we are going to introduce a suitable form of subspace of a Jäger-Šsostak's $I$-fuzzy topological space.

Let $A \in[0,1]^{X}$ and $B \in \mathcal{F}_{X}(A), i_{B}: B \rightarrow A$ such that $i_{B}(C)=C$ (that is, $\left.i_{B}=i d_{X} \mid B\right)$. If $(A, \tau)$ is a JSI-fts, we call $\tau_{B}^{c}: \mathcal{F}_{X}(B) \rightarrow I$ such that $\forall K \in \mathcal{F}_{X}(B)$,

$$
\tau_{B}^{c}(K)=\bigvee_{\langle B(H \wedge B)=K} \tau\left(\left\langle{ }^{A} H\right)=\bigvee_{H \wedge B=\left\langle{ }^{B} K\right.} \tau\left(\left\langle{ }^{A} H\right)\right.\right.
$$

the Jäger-Šostak's $I$-fuzzy subspace topology on B induced by $\tau$, and call the pair $\left(B, \tau_{B}^{c}\right)$ the Jäger-Šostak's $I$-fuzzy subspace of $(A, \tau)$. When $\tau$ is a fuzzy topology on $\mathrm{A}$ in the sense of G. Jäger [6], the definition is identified with the definition of fuzzy subspace of G. Jäger [6]. Actually, this subspace concept was first used in Liu and Luo [7] but was so far not reached general interest in $I$-fuzzy setting.

Proposition 2.7. For any JSI-fts $(A, \tau)$, we have $\left(\tau_{B}^{c}\right)_{C}^{c}=\tau_{C}^{c}$ for $C \leq B \leq$ A.

Proof. Let us confirm that for each $H \in \mathcal{F}_{X}(C),\left(\tau_{B}^{c}\right)_{C}^{c}(H)=\tau_{C}^{c}(H)$ is true, and hence the conclusion is deduced from the arbitrariness of $\mathrm{H}$.

First of all, for each $H \in \mathcal{F}_{X}(C)$ we have that

$$
\begin{aligned}
& \left(\tau_{B}^{c}\right)_{C}^{c}(H)=\bigvee_{\substack{G \leq B \\
G \wedge C=\left\langle{ }^{C} H\right.}} \tau_{B}^{c}\left(\left\langle{ }^{B} G\right)\right. \\
& =\bigvee_{\substack{G \leq B \\
G \wedge C=\left\langle{ }^{C} H\right.}} \bigvee_{\substack{K \leq A \\
K \wedge B}} \tau\left(\left\langle\left\langle^ { B } \left\langle^{B} G\right.\right.\right.\right. \\
& =\bigvee_{G \leq B} \bigvee_{K \leq A} \tau\left(\left\langle{ }^{A} K\right)\right. \\
& G \wedge C=\left\langle{ }^{C} H \quad K \wedge B=G\right. \\
& \leq \quad \bigvee \quad \tau\left(\left\langle{ }^{A} R\right)\right. \\
& \begin{array}{c}
R \leq A \\
R \wedge C=\left\langle{ }^{C} H\right.
\end{array} \\
& =\tau_{C}^{c}(H) \text {. }
\end{aligned}
$$


because for each $G \leq B$ with $G \wedge C=\left\langle{ }^{C} H\right.$ and each $K \leq A$ with $K \wedge B=G$, we have

$$
K \wedge C=K \wedge B \wedge C=G \wedge C=\left\langle{ }^{C} H .\right.
$$

On the other hand, when letting $G:=R \wedge B$ for $R \leq A$ with $R \wedge C=$ $\left\langle{ }^{C} H\right.$, we have

$$
G \wedge C=R \wedge B \wedge C=R \wedge C=\left\langle{ }^{C} H\right.
$$

and R satisfies $R \wedge B=G$ already. Thus

$$
\begin{aligned}
& \tau_{C}^{c}(H)=\bigvee_{\substack{R \leq A \\
R \wedge C=\left\langle{ }^{C} H\right.}} \tau\left(\left\langle{ }^{A} R\right)\right. \\
& \leq \bigvee_{\substack{G \leq B \\
G \wedge C=\left\langle{ }^{C} H\right.}} \bigvee_{\substack{K \leq A \\
K \wedge B=G}}^{\bigvee} \tau\left(\left\langle{ }^{A} K\right)\right. \\
& =\left(\tau_{B}^{c}\right)_{C}^{c}(H) \text {. }
\end{aligned}
$$

Therefore, it follows that for each $H \leq C$ (or $\left.H \in \mathcal{F}_{X}(C)\right),\left(\tau_{B}^{c}\right)_{C}^{c}(H)=$ $\tau_{C}^{c}(H)$.

Proposition 2.8. For any $(A, \tau)$ JSI-fts and any fuzzy subset $B \in I^{X}$ with $B \leq A$, it holds

$$
\tau_{B}^{c}\left(\left\langle{ }^{B} K\right)=\bigvee_{H \wedge B=K} \tau\left(\left\langle{ }^{A} H\right),\right.\right.
$$

for each $K \leq B$.

Proof. This is obvious by the definition of $\tau_{B}^{c}$.

According to Remarks 2.2, when a $\mathcal{T}$ is a (general) fuzzy topology on a fuzzy subset $A$ and we identify the Jäger-Šostak's $I$-fuzzy topology $\tau_{\mathcal{T}}$ induced by $\mathcal{T}$ with $\mathcal{T}$, then the results stated in [6] can be described as follows.

Corollary 2.9. Let $(A, \mathcal{T})$ be a (general) fuzzy topological space and $C \leq B \leq A$. Then for each $E \leq C$, it holds that $\left(\mathcal{T}_{B}^{c}\right)_{C}^{c}(E)=1 \Leftrightarrow \mathcal{T}_{C}^{c}(E)=$ 1 . 
Corollary 2.10. Let $(A, \mathcal{T})$ be a (general) fuzzy topological space and $B \leq A$. We then have for each $M \leq B, \mathcal{T}_{B}^{c}\left(\left\langle{ }^{B} M\right)=1\right.$ if and only if there exists a $H \leq A$ such that $\mathcal{T}\left(\left\langle{ }^{A} H\right)=1\right.$ and $M=B \wedge H$.

For an $I$-fuzzy topology $\mathcal{S}$ on a set $X$ in the sense of Höhle and Šstak [5], there exists a good way defining $I$-fuzzy subspace topology on a crisp subset $Y \subseteq X[2,10]$. In the following, we hope to show this kind of $I$-fuzzy subspace topology could be described as a special case of Jäger-Šsostak's $I$ fuzzy sub-topology of the paper. Before we state the results we quote the definition below.

Definition 2.11 (Höhle and Šostack [5]). An I-fuzzy topology on a set $X$ is a map $\mathcal{S}: I^{X} \rightarrow I$ such that

(I-FO1) $S(\lambda)=1$ for every constant fuzzy subset $\lambda: X \rightarrow I$;

$(\mathrm{I}-\mathrm{FO} 2) \mathcal{S}(U \wedge V) \geq \mathcal{S}(U) \wedge \mathcal{S}(V), \forall U, V \in I^{X}$

$(\mathrm{I}-\mathrm{FO} 3) \mathcal{S}\left(\bigvee_{j \in J} U_{j}\right) \geq \bigwedge_{j \in J} \mathcal{S}\left(U_{j}\right), \forall j \in J, U_{j} \in I^{X}$.

If $\mathcal{S}$ is an $I$-fuzzy topology on $X$, then we say that $\left(I^{X}, \mathcal{S}\right)$ is an $I$-fuzzy topological space ( $I$-fts, in short).

Obviously, every $I$-fuzzy topological space $\left(I^{X}, \mathcal{S}\right)$ can be identified as a Jäger-Šsostak's $I$-fuzzy topological space $\left(1_{X}, \mathcal{S}\right)$ if necessary.

In [7] and later [2], a concept of $I$-fuzzy sub-topology was proposed, in details, for a crisp subset $Y \subseteq X$, an $I$-fuzzy sub-topology $\mathcal{S}_{Y}: I^{Y} \rightarrow I$ on $Y$ induced by $\mathcal{S}$ is defined as follows: for each $U \in I^{Y}$,

$$
\mathcal{S}_{Y}(U)=\bigvee\left\{\mathcal{S}(V): V \in I^{X}, V \mid Y=U\right\}
$$

and $\left(I^{Y}, \mathcal{S}_{Y}\right)$ is called the $I$-fuzzy topological subspace of $\left(I^{X}, \mathcal{S}\right)$.

Proposition 2.12. Let $\left(I^{X}, \mathcal{S}\right)$ be an $I$-fts and $B=\mathbf{1}_{Y}$, where $Y \subseteq X$, a crisp subset of X. Then $\mathcal{S}_{B}^{c}=\mathcal{S}_{Y}$.

Proof. Note that we identified $\mathcal{F}_{X}(B)$ with $\mathcal{F}_{Y}(B)$. The equality $\mathcal{S}_{B}^{c}=\mathcal{S}_{Y}$ 
is obtained by the following formulas, $\forall H \in \mathcal{F}_{Y}(B)$,

$$
\begin{aligned}
\mathcal{S}_{B}^{c}(H) & =\bigvee_{G \wedge B=\langle B} \mathcal{S}\left(\left\langle{ }^{1}{ }^{X} G\right)=\bigvee_{\left(\left\langle{ }^{1} X G\right) \wedge B=\langle B\right.} \mathcal{S}(G)\right. \\
& =\bigvee_{G \mid Y=H} \mathcal{S}(G)=\left(\mathcal{S}_{Y}\right)(H) .
\end{aligned}
$$

because $G \mid Y=H \Leftrightarrow\left(\left\langle{ }^{1}{ }_{X} G\right) \wedge B=\left\langle{ }^{B} H\right.\right.$, for each $G \in \mathcal{F}_{X}\left(\mathbf{1}_{X}\right)$, $H \in \mathcal{F}_{Y}(B)$.

Remark 2.13. Proposition 3.4 shows that for a crisp subset $B=\mathbf{1}^{Y}$ of an $I$-fts $\left(I^{X}, \mathcal{S}\right)$, the subspace topologies $\mathcal{S}_{B}^{c}$ and $\mathcal{S}_{Y}[2,10]$ coincide.

\section{The degree of $I$-fuzzy connectedness of a fuzzy set}

In classical topology, there are many ways to describe the definition of connectedness. For example, no existing separation, each continuous function into the discrete two points space being constant, K. Fan theorem's way, etc. In [6], G. Jäger gave a definition of connectedness of a fuzzy subset in a (general) fuzzy topological space similar to the one in classical topology, i.e., a topological space is connected iff each continuous function into discrete two points space is constant, and it was proved by Jäger, that this kind of connectedness of a fuzzy subset is an absolute property. The definition adopted in [6] was proved to be equivalent to $\mathrm{Pu}$ and Liu's definition [9] even for fuzzy subsets. Jäger's manner defining connectedness of a fuzzy subset also can be found in Lowen and Srivastava [8] in the case that the fuzzy subset is the whole space.

The degree of connectedness of fuzzy subsets presented here in JägerŠostak's $I$-fuzzy topological spaces will be introduced in this section. If the degree of connectedness of a fuzzy subset is the designed truth value 1 , we then go back to Jäger's definition [6] in two-valued logic. We will prove here our general $I$-fuzzy degree of connectedness is absolute.

In the following, we call $H_{t}(A):=\bigvee_{x \in X} A(x)$ the height of $A$ for each $A \in[0,1]^{X}$ and for $\mathbf{2}=\{0,1\}, 0<\alpha \leq 1$, define $\mathbf{2}_{\alpha}=\alpha \wedge 1_{\mathbf{2}}$, where $\mathbf{1}_{\mathbf{2}}$ is the characteristic function of $\mathbf{2}$. The discrete Jäger-Šstak's $I$-fuzzy topology on $\mathbf{2}_{\alpha}$ is denoted by $T_{\alpha}$, which means that $T_{\alpha}(B)=1$ for all 
$B \leq \mathbf{2}_{\alpha}$. Furthermore, for $A \in I^{X}, B \in I^{Y}$, we call a fuzzy mapping $g=f \mid A: A \rightarrow B$ constant iff $f \mid A_{0}: A_{0} \rightarrow Y$ is constant. The set of all fuzzy mappings from A to B which isn't constant, is denoted by $\operatorname{Sur}\left(A_{0}, Y\right)$.

Definition 3.1. Let $\sum$ denote the class of all Jäger-Šsostak's $I$-fuzzy topological spaces. A mapping $\operatorname{Con}_{G}: \sum \rightarrow I$, called a general $I$-fuzzy degree of connectedness, is given as follows:

$$
\forall(A, \tau) \in \sum, \operatorname{Con}_{G}(A, \tau)=1-\bigvee_{g \in \operatorname{Sur}\left(A_{0}, \mathbf{2}\right)} \bigwedge_{C \leq \mathbf{2}_{\alpha}} \tau\left(\left\langle{ }^{A} g^{-1}(C)\right),\right.
$$

where $\alpha=H_{t}(A)$.

Corollary 3.2. Let $(A, \tau)$ be JSI-fts and $\mathrm{g}$ be a fuzzy mapping from A to $\mathbf{2}_{H_{t}(A)}$. Then $c(g)=\bigwedge_{D \leq \mathbf{2}_{H_{t}(A)}} \tau\left(\left\langle{ }^{A} g^{-1}(D)\right)\right.$.

Proof. By the definition above,

$$
\begin{gathered}
c(g)=\bigwedge_{D \leq \mathbf{2}_{H_{t}(A)}} \min \left\{1,1-T_{H_{t}(A)}\left(\left\langle\mathbf{2}_{H_{t}(A)}(D)\right)+\tau\left(\left\langle{ }^{A} g^{-1}(D)\right)\right\}\right.\right. \\
=\bigwedge_{D \leq \mathbf{2}_{H_{t}(A)}} \tau\left(\left\langle{ }^{A} g^{-1}(D)\right),\right.
\end{gathered}
$$

since $T_{H_{t}(A)}\left(\left\langle\mathbf{2}_{H_{t}(A)}(D)\right)=1\right.$ always.

Let us give the first characteristic theorem of the general $I$-fuzzy degree of connectedness as follows.

Theorem 3.3. Let $(A, \tau)$ be a JSI-fts. Then

$$
\operatorname{Con}_{G}(A, \tau)=1-\bigvee_{\emptyset \neq E \subset A_{0}} \tau\left(\langle { } ^ { A } ( \mathbf { 1 } _ { E } \wedge A ) ) \wedge \tau \left(\left\langle{ }^{A}\left(\mathbf{1}_{A_{0}-E} \wedge A\right)\right)\right.\right.
$$

Proof. According to the definition of $\operatorname{con}_{G}(A, \tau)$, we need to prove

$$
\bigvee_{g \in \operatorname{Sur}\left(A_{0}, \mathbf{2}\right)} \bigwedge_{C \leq \mathbf{2}_{H_{t}(A)}}\left(\tau\left\langle^{A}\left(g^{-1}(C)\right)\right)=\bigvee_{\emptyset \neq E \subset A_{0}} \tau\left(\langle { } ^ { A } ( \mathbf { 1 } _ { E } \wedge A ) ) \wedge \tau \left(\left\langle{ }^{A}\left(\mathbf{1}_{A_{0}-E} \wedge A\right)\right)\right.\right.\right.
$$


To complete the target here, let us assume that

$$
\alpha=\bigvee_{g \in \operatorname{Sur}\left(A_{0}, \mathbf{2}\right)} \bigwedge_{C \leq \mathbf{2}_{H_{t}(A)}} \tau\left(\left\langle{ }^{A} g^{-1}(C)\right)\right.
$$

and

$$
\beta=\bigvee_{\phi \neq E \subset A_{0}} \tau\left(\langle { } ^ { A } ( \mathbf { 1 } _ { E } \wedge A ) ) \wedge \tau \left(\left\langle{ }^{A}\left(\mathbf{1}_{A_{0}-E} \wedge A\right)\right)\right.\right.
$$

in the present time. Thus our target change to prove $\alpha=\beta$. The strategy here from is to show both $\alpha \leq \beta$ and $\beta \leq \alpha$. Let $\gamma$ be any real number such that $0 \leq \gamma<\beta$. According to the definition of $\beta$ there is a non-void proper subset $\mathrm{E}$ of $A_{0}$ such that

$$
\tau\left(\left\langle{ }^{A}\left(\mathbf{1}_{E} \wedge A\right)\right)>\gamma\right.
$$

and

$$
\tau\left(\left\langle{ }^{A}\left(\mathbf{1}_{A_{0}-E} \wedge A\right)\right)>\gamma\right.
$$

Now, we define $f: X \rightarrow \mathbf{2}$ by $f(x)=0$ if $x \in E$ and $f(x)=1$ else. Then $g_{*}:=f \mid A:(A, \tau) \rightarrow\left(\mathbf{2}_{H_{t}(A)}, T_{H_{t}(A)}\right)$ is continuous and surjective. Then for $t: 0 \leq t \leq H_{t}(A)$, we have

$$
\begin{aligned}
& \tau\left(\left\langle^{A}\left(g_{*}^{-1}\left(\left\langle^{A} t \wedge \mathbf{1}_{\{0\}}\right)\right)\right)=\tau\left(\left\langle^{A}\left(A \wedge f^{-1}\left(t \wedge \mathbf{1}_{\{0\}}\right)\right)\right)\right.\right.\right. \\
& =\tau\left(\left\langle^{A}\left(A \wedge t \wedge \mathbf{1}_{f^{-1}(\{0\})}\right)\right)\right. \\
& =\tau\left(\left\langle^{A}\left(A \wedge t \wedge \mathbf{1}_{E}\right)\right)\right. \\
& =\tau\left(\left\langle{ }^{A}\left(A \wedge t \wedge A \wedge \mathbf{1}_{E}\right)\right)\right. \\
& =\tau\left(\left\langle{ }^{A}(A \wedge t) \vee\left\langle{ }^{A}\left(A \wedge \mathbf{1}_{E}\right)\right)\right.\right. \\
& \geq \tau\left(\langle ^ { A } ( A \wedge \mathbf { 1 } _ { E } ) ) \wedge \tau \left(\left\langle^{A}(A \wedge t)\right)\right.\right. \\
& =\tau\left(\left\langle{ }^{A}\left(A \wedge \mathbf{1}_{E}\right)\right)\right. \\
& >\gamma
\end{aligned}
$$


since $\tau\left(\left\langle{ }^{A}(t \wedge A)\right)=1\right.$. Similarly, we see that $\tau\left(\left\langle{ }^{A}\left(g_{*}^{-1}\left(\left\langle{ }^{A} s \wedge \mathbf{1}_{\{1\}}\right)\right)\right)>\right.\right.$

$\gamma$ for each s with $0 \leq s \leq H_{t}(A)$. Everyone know that there are $t_{D}, s_{C}$ with the property of $0 \leq t_{D}, s_{C} \leq H_{t}(B)$ so that $D=t_{D} \wedge \mathbf{1}_{\{0\}} \vee s_{C} \wedge \mathbf{1}_{\{1\}}$ for any $D \leq \mathbf{2}_{H_{t}(A)}$. Thus using $g_{*}$, it holds

$$
\begin{aligned}
\alpha & =\bigwedge_{g \in \operatorname{Sur}\left(A_{0}, \mathbf{2}\right)} \bigwedge_{D \leq \mathbf{2}_{H_{t}(A)}} \tau\left(\left\langle{ }^{A} g^{-1}(D)\right)\right. \\
& \geq \bigwedge_{D \leq \mathbf{2}_{H_{t}(A)}} \tau\left(\left\langle{ }^{A} g_{*}^{-1}(D)\right)\right. \\
& \geq \bigwedge_{\substack{D \leq \mathbf{2}_{H_{t}(A)} \\
C \leq \mathbf{2}_{H_{t}(A)}}} \tau\left(\langle { } ^ { A } g _ { * } ^ { - 1 } ( t _ { D } \mathbf { 1 } _ { \{ 0 \} } ) ) \wedge \tau \left(\left\langle{ }^{A} g_{*}^{-1}\left(s_{C} \mathbf{1}_{\{1\}}\right)\right)\right.\right. \\
& \geq \gamma .
\end{aligned}
$$

By the arbitrariness of $\gamma$, we get $\beta \leq \alpha$.

Conversely, let $\gamma$ be any real number with $0 \leq \gamma<\alpha$ now. It follows from the definition of $\alpha$ that there exists a mapping $g \in \operatorname{Sur}\left(A_{0}, \mathbf{2}\right)$, and here $g=f \mid A$ such that there exist two non-void disjoint subsets $E_{1}$ and $F_{1}$ of $A_{0}$ with the properties of $f\left(E_{1}\right)=\{0\}, f\left(F_{1}\right)=\{1\}, E_{1} \cup F_{1}=A_{0}$. Moreover, for each $D \leq \mathbf{2}_{H_{t}(A)}$, it holds $\tau\left(\left\langle{ }^{A} g^{-1}(D)\right)>\gamma\right.$. Thus we easily see that

$$
\begin{aligned}
\beta & =\bigvee_{\phi \neq E \subset A_{0}} \tau\left(\langle { } ^ { A } ( \mathbf { 1 } _ { E } \wedge A ) ) \wedge \tau \left(\left\langle{ }^{A}\left(\mathbf{1}_{A_{0}-E} \wedge A\right)\right)\right.\right. \\
& \geq \tau\left(\langle { } ^ { A } ( \mathbf { 1 } _ { E _ { 1 } } \wedge A ) ) \wedge \tau \left(\left\langle{ }^{A}\left(\mathbf{1}_{F_{1}} \wedge A\right)\right)\right.\right. \\
& =\tau\left(\langle { } ^ { A } g ^ { - 1 } ( H _ { t } ( A ) \wedge \mathbf { 1 } _ { \{ 0 \} } ) ) \wedge \tau \left(\left\langle{ }^{A} g^{-1}\left(H_{t}(A) \wedge \mathbf{1}_{\{1\}}\right)\right)\right.\right. \\
& >\gamma
\end{aligned}
$$

Therefore, $\alpha \leq \beta$ holds from the arbitrariness of $\gamma$.

Corollary 3.4. For a JSI-fts $(A, \tau)$,

$$
\operatorname{Con}_{G}(A, \tau)=1-\bigvee_{\emptyset \neq E \subset A_{0}} \tau\left(\mathbf{1}_{E} \wedge A\right) \wedge \tau\left(\mathbf{1}_{A_{0}-E} \wedge A\right) .
$$


Proof. This is obvious by Theorem 3.3, since $\left\langle{ }^{A}\left(\mathbf{1}_{E} \wedge A\right)=\mathbf{1}_{A_{0}-E} \wedge A\right.$ and $\left\langle{ }^{A}\left(\mathbf{1}_{A_{0}-E} \wedge A\right)=\mathbf{1}_{E} \wedge A\right.$.

Corollary 3.5. Let $\left(1_{X}, \tau\right)$ is a JSI-fts, i.e., $\tau$ is an $I$-fuzzy topology on $X$ (see Definition 2.11). Then it holds $\operatorname{Con}\left(I^{X}, \tau\right)=\operatorname{Con}_{G}\left(\mathbf{1}_{X}, \tau\right)$, where

$$
\operatorname{Con}\left(I^{X}, \tau\right)=1-\bigvee_{(B, C) \in \mathcal{D}} \tau(B) \wedge \tau(C)
$$

defined by Fang and Yue [3] and its $\mathcal{D}=\left\{(B, C) \in I^{X} \times I^{X} \mid B \neq \mathbf{0}_{X}, C \neq\right.$ $\mathbf{0}_{X}, B \vee C=\mathbf{1}_{X}$ and $\left.B \wedge C=\mathbf{0}_{X}\right\}$.

Proof. Note that for each pair $(B, C) \in I^{X} \times I^{X}$, it holds that $(B, C) \in \mathcal{D}$ if and only if $B=\mathbf{1}_{B_{0}}, C=\mathbf{1}_{C_{0}}, B_{0} \cup C_{0}=X$ and $B_{0} \cap C_{0}=\emptyset$. Thus using Corollary 3.4, we have

$$
\begin{aligned}
\operatorname{Con}_{G}\left(\mathbf{1}^{X}, \tau\right) & =1-\bigvee_{\emptyset \neq E \subset X} \tau\left(\mathbf{1}_{E} \wedge \mathbf{1}_{X}\right) \wedge \tau\left(\mathbf{1}_{X-E} \wedge \mathbf{1}_{X}\right) \\
& =1-\bigvee_{(B, C) \in \mathcal{D}} \tau(B) \wedge \tau(C) .
\end{aligned}
$$

Remark 3.6. By Corollary 3.5, we have generalized $I$-fuzzy connection proposed in [3] to more general form, that is , the general $I$-fuzzy degree of connectedness for any fuzzy subsets.

As pointed out at the beginning of the section, there are many ways to describe the definition of connectedness. Now we try to characterize the general $I$-fuzzy degree of connectedness by the ideas of separation. Now, a definition of separation in fuzzy setting is needed.

Definition 3.7. Let $(A, \tau)$ be a JSI-fts. A mapping $\mathcal{S}_{G}:\left(\mathcal{F}_{X}(A)-\right.$ $\left.\left\{\mathbf{0}_{X}\right\}\right) \times\left(\mathcal{F}_{X}(A)-\left\{\mathbf{0}_{X}\right\}\right) \rightarrow I$, called a general $I$-fuzzy degree of separation, is given as follows:

$$
\forall M, N \in \mathcal{F}_{X}(A)-\left\{\mathbf{0}_{X}\right\}, \quad \mathcal{S}_{G}(M, N)=\underset{\substack{(H, K) \geq(M, N) \\ H \wedge N=K \wedge M=\mathbf{0}_{X}}}{\bigvee} \tau\left(\langle { } ^ { A } H ) \wedge \tau \left(\left\langle{ }^{A} K\right)\right.\right.
$$


Following the definition above, the characteristic theorem of general $I$ fuzzy degree of connectedness using the general $I$-fuzzy degree of separation is obtained.

Theorem 3.8. Let $(A, \tau)$ be a JSI-fts, $D \in \mathcal{F}_{X}(A)$. Then

$$
\operatorname{Con}_{G}\left(D, \tau_{D}^{c}\right)=1-\bigvee_{M \vee N=D} \mathcal{S}(M, N)
$$

Especially when $D=A$, it holds

$$
\operatorname{Con}_{G}(A, \tau)=1-\bigvee_{M \vee N=A} \mathcal{S}(M, N) .
$$

Proof. By Theorem 3.3,

$$
\operatorname{Con}_{G}\left(D, \tau_{D}^{c}\right)=1-\bigvee_{\emptyset \neq E \subset D_{0}} \tau_{D}^{c}\left(\langle { } ^ { D } \mathbf { 1 } _ { E } \wedge D ) \wedge \tau _ { D } ^ { c } \left(\left\langle{ }^{D} \mathbf{1}_{D_{0}-E} \wedge D\right)\right.\right.
$$

It suffices to show that

$$
\bigvee_{\emptyset \neq E \subset D_{0}} \tau_{D}^{c}\left(\langle { } ^ { D } \mathbf { 1 } _ { E } \wedge D ) \wedge \tau _ { D } ^ { c } \left(\left\langle\left\langle^{D} \mathbf{1}_{D_{0}-E} \wedge D\right)=\bigvee_{M \vee N=D} \mathcal{S}(M, N)\right.\right.\right.
$$

Now letting $\gamma$ be any positive real number with the property of

$$
\gamma<\bigvee_{\emptyset \neq E \subset D_{0}} \tau_{D}^{c}\left(\langle ^ { D } ( \mathbf { 1 } _ { E } \wedge D ) ) \wedge \tau _ { D } ^ { c } \left(\left\langle^{D}\left(\mathbf{1}_{D_{0}-E} \wedge D\right)\right),\right.\right.
$$

then there exists a non-void proper subset $E$ of $D_{0}$ such that

$$
\gamma<\tau_{D}^{c}\left(\left\langle{ }^{D}\left(\mathbf{1}_{E} \wedge D\right)\right) \text { and } \gamma<\tau_{D}^{c}\left(\left\langle{ }^{D}\left(\mathbf{1}_{D_{0}-E} \wedge D\right)\right) .\right.\right.
$$

Since

$$
\tau_{D}^{c}\left(\left\langle^{D}\left(\mathbf{1}_{E} \wedge D\right)\right)=\bigvee_{R \wedge D=\mathbf{1}_{E} \wedge D} \tau\left(\left\langle{ }^{A} R\right)\right.\right.
$$

and

$$
\tau_{D}^{c}\left(\left\langle{ }^{D}\left(\mathbf{1}_{D_{0}-E} \wedge D\right)\right)=\bigvee_{S \wedge D=\mathbf{1}_{D_{0}-E} \wedge D} \tau\left(\left\langle{ }^{A} S\right),\right.\right.
$$

it follows that there exist $R^{*}, S^{*} \in \mathcal{F}_{X}(A)$ such that $R^{*} \wedge D=\mathbf{1}_{E} \wedge D$, $S^{*} \wedge D=\mathbf{1}_{D_{0}-E} \wedge D, \gamma<\tau\left(\left\langle{ }^{A} R^{*}\right)\right.$ and $\gamma<\tau\left(\left\langle{ }^{A} S^{*}\right)\right.$. Taking $M^{*}=\mathbf{1}_{E} \wedge D$ 
and $N^{*}=\mathbf{1}_{D_{0}-E} \wedge D$, we easily obtain that $M^{*} \leq R^{*}, N^{*} \leq S^{*}, M^{*} \vee N^{*}=$ $D$, and moreover,

$$
R^{*} \wedge N^{*}=R^{*} \wedge D \wedge \mathbf{1}_{D_{0}-E}=\mathbf{1}_{E} \wedge \mathbf{1}_{D_{0}-E} \wedge D=\mathbf{0}_{X}
$$

and

$$
S^{*} \wedge M^{*}=S^{*} \wedge D \wedge \mathbf{1}_{E}=\mathbf{1}_{E} \wedge \mathbf{1}_{D_{0}-E} \wedge D=\mathbf{0}_{X}
$$

Thus,

$$
\begin{aligned}
\bigvee_{M \vee N=D} \mathcal{S}(M, N) & \geq \mathcal{S}\left(M^{*}, N^{*}\right) \\
& \geq \tau\left(\langle { } ^ { A } R ^ { * } ) \wedge \tau \left(\left\langle{ }^{A} S^{*}\right)\right.\right. \\
& \geq \gamma .
\end{aligned}
$$

It follows from the arbitrariness of $\gamma$, that

$$
\bigvee_{\emptyset \neq E \subset D_{0}} \tau_{D}^{c}\left(\langle { } ^ { D } \mathbf { 1 } _ { E } \wedge D ) \wedge \tau _ { D } ^ { c } \left(\left\langle{ }^{D} \mathbf{1}_{D_{0}-E} \wedge D\right) \leq \bigvee_{M \vee N=D} \mathcal{S}(M, N)\right.\right.
$$

Conversely, let $\gamma$ be any positive real number so that

$$
\gamma<\bigvee_{M \vee N=D} \mathcal{S}(M, N)=\bigvee_{M \vee N=D} \bigvee_{\substack{(H, K) \geq(M, N) \\ H \wedge N=K \wedge M=\mathbf{0}_{X}}} \tau\left(\langle { } ^ { A } H ) \wedge \tau \left(\left\langle{ }^{A} K\right)\right.\right.
$$

Then it follows that there exist $M, N \in \mathcal{F}_{X}(D)-\left\{\mathbf{0}_{X}\right\}$ and $H, K \in$ $\mathcal{F}_{X}(A)$, such that $M \vee N=D, \quad(H, K) \geq(M, N), H \wedge N=K \wedge M=$ $\mathbf{0}_{X}, \gamma<\tau\left(\left\langle{ }^{A} H\right)\right.$ and $\gamma<\tau\left(\left\langle{ }^{A} K\right)\right.$. Furthermore, we obtain that $M_{0} \cup N_{0}=$ $D_{0}, M_{0} \cap N_{0}=\emptyset$,

$$
K \wedge D=K \wedge(M \vee N)=K \wedge N=N=\mathbf{1}_{N_{0}} \wedge D
$$

and

$$
H \wedge D=H \wedge(M \vee N)=H \wedge M=M=\mathbf{1}_{M_{0}} \wedge D
$$

It concludes that

$$
\bigvee_{\emptyset \neq E \subset D_{0}} \tau_{D}^{c}\left(\langle ^ { D } ( \mathbf { 1 } _ { E } \wedge D ) ) \wedge \tau _ { D } ^ { c } \left(\left\langle^{D}\left(\mathbf{1}_{D_{0}-E} \wedge D\right)\right)\right.\right.
$$




$$
\begin{aligned}
& \geq \tau_{D}^{c}\left(\langle { } ^ { D } ( \mathbf { 1 } _ { N _ { 0 } } \wedge D ) ) \wedge \tau _ { D } ^ { c } \left(\left\langle{ }^{D}\left(\mathbf{1}_{M_{0}} \wedge D\right)\right)\right.\right. \\
& =\left[\bigvee _ { R \wedge D = \mathbf { 1 } _ { N _ { 0 } } \wedge D } \tau ( \langle { } ^ { A } R ) ] \wedge \left[\bigvee_{S \wedge D=\mathbf{1}_{M_{0}} \wedge D} \tau\left(\left\langle{ }^{A} S\right)\right]\right.\right. \\
& \geq \tau\left(\langle { } ^ { A } H ) \wedge \tau \left(\left\langle{ }^{A} K\right)\right.\right. \\
& >\gamma .
\end{aligned}
$$

Therefore,

$$
\bigvee_{M \vee N=D} \mathcal{S}(M, N) \leq \bigvee_{\emptyset \neq E \subset D_{0}} \tau_{D}^{c}\left(\langle { } ^ { D } \mathbf { 1 } _ { E } \wedge D ) \wedge \tau _ { D } ^ { c } \left(\left\langle{ }^{D} \mathbf{1}_{D_{0}-E} \wedge D\right)\right.\right.
$$

Finally, we complete the proofs by the two inequalities (3.1) and (3.2) above.

At the end of the section, we want to study the absolute property of the general $I$-fuzzy degree of connectedness defined in the paper. Recall that we call a property $\mathrm{P}$ is absolute in classical topology if for all subspaces $Z \subseteq Y \subseteq X$ of a topological space $(X, T), Z$ has the property $\mathrm{P}$ as a subspace of $Y$ if and only if $\mathrm{Z}$ has the property $\mathrm{P}$ as a subspace of $\mathrm{X}$. In $I$-fuzzy setting we propose a definition as follows: a degree mapping $\Gamma$ describing a property $\mathrm{P}$ is said to be absolute if and only if for all subspaces $C \leq B \leq A$ of a Jäger-Šostak's $I$-fuzzy topological space $(A, \tau)$, the true value $\Gamma(C)$ having property $\mathrm{P}$ as a subspace of $B$ is equal to the true value $\Gamma(C)$ having property $\mathrm{P}$ as a subspace of $A$. In this way, we have

Theorem 3.9. The general $I$-fuzzy degree of connectedness is absolute.

Proof. Let $(A, \tau)$ be a JSI-fts and $C \leq B \leq A$. Thus there are two topologies $\left(\tau_{B}^{c}\right)_{C}^{c}$ and $\tau_{C}^{c}$ on C. By proposition 2.7 , we know that $\left(\tau_{B}^{c}\right)_{C}^{c}=\tau_{C}^{c}$. It follows from the definition of the general $I$-fuzzy degree of connectedness that $\operatorname{Con}_{G}\left(C, \tau_{C}^{c}\right)=\operatorname{Con}_{G}\left(C,\left(\tau_{B}^{c}\right)_{C}^{c}\right)$, that is, the degree mapping $C o n_{G}$ stating general $I$-fuzzy degree of connectedness is absolute, as desired. 


\section{Conclusions.}

In this paper, we propose a new concept of Jäger-Šostak's I-fuzzy topological space for any fuzzy subset in place of a whole space. Obviously, this is a new kind of fuzzy topology, namely Jäger-Šostak's I-fuzzy topology with the fixed basis $L=I$, the real unit interval. Some of basic concepts are established, such as subspaces and suitable morphisms. In the framework of Jäger-Šostak's $I$-fuzzy topology, we research the general $I$-fuzzy degree of connectedness of a fuzzy subset in Jäger-Šsostak's $I$-fuzzy topology, and its characteristic theorems are obtained. Moreover, we show that the general $I$-fuzzy degree of connectedness defined in the paper is absolute, which reflect the concept of subspace in the paper is rational. We think there are a lot of questions, such as Neighborhood systems, Moore Smith's convergence, separation axioms, compactness, etc., needed to be settled. We will exploit them in our following works.

\section{References}

[1] Chang C. L. Fuzzy topological space, J. Math. Anal. Appl. 24, pp. 182-190, (1968).

[2] Fang J. M., Yue Y. L, Base and Subbase in $I$-fuzzy topological space, Journal of Mathematical Research and Exposition, 26(1), pp. 89-95, (2006).

[3] Fang J. M., Yue Y. L. Generalized connectivity, Proyecciones Journal of Mathematics 25(2), pp. 191-203, (2006).

[4] Fang J. M., Yue Y L. K. Fan's theorem in fuzzifying topology, Information Sciences 162, pp. 139-146, (2004).

[5] Höhle U, Šostak A P. Axiomatic foundations of fixed basis fuzzy topology, Chapter 3 in: U. Höhle, S. E. Rodaubaugh (Eds.), Mathematics of Fuzzy Sets: Logic, Topology and Measure Theory, Kluwer Academic Publishers, Boston/Dordrecht/London, pp. 123-272, (1999).

[6] Jäger G. Compactness and connectedness as absolute properties in fuzzy topological spaces, Fuzzy sets and Systems 94, pp. 405-401, (1998). 
[7] Liu Y. M., Luo M. K. Induced spaces and fuzzy Stone-cech compactifications, Scientia Sinica (A) 30, pp. 1034-1044, (1987).

[8] Lowen R., Srivastava A. K., On Press'Connectedness concept in Fts, Fuzzy Set and Systems 47, pp. 99-104, (1992).

[9] Pu B. M., Liu Y. M. Fuzzy topology I-Neighborhood structure of a fuzzy point and Moon-Smith convergence, J. Math. Anal. Appl. 76, pp. 571-599, (1980).

[10] Werner P. Subspaces of smooth fuzzy topologies and initial smooth fuzzy structure, Fuzzy Sets and Systems 104, pp. 423-433, (1999).

[11] Ying M. S., A new approach to fuzzy topology (II), Fuzzy sets and systems 47, pp. 221-232, (1992).

\section{Fang Jinming}

Department of Mathematics, Ocean University of China, 23, Hong Kong Road,

Qing Dao 266071,

P. R. China

China

e-mail : gymaizw63.com

and

\section{Guo Yuanmei}

Department of Mathematics, Ocean University of China, 23, Hong Kong Road, Qing Dao 266071, P. R. China

China

e-mail : gymaizw63.com 